\title{
Erratum to: Increase male genital diseases morbidity linked to informal electronic waste recycling in Guiyu, China
}

\author{
Xijin Xu • Yuling Zhang • Taofeek Akangbe Yekeen • \\ Yan Li $\cdot$ Bingrong Zhuang $\cdot$ Xia Huo
}

Published online: 14 June 2014

(C) Springer-Verlag Berlin Heidelberg 2014

\section{Erratum to: Environ Sci Pollut Res (2014) 21:3540-3545 DOI 10.1007/s11356-013-2289-2}

We wish to correct a mistake in Table 1 of this article in which the years in the first column ought to be in chronological order i.e. 2001, 2002, 2003, to 2009 instead of 2001, 2001, 2003 to 2009.

Table 1 Composition of morbidity of male genital diseases between Guiyu and control

\begin{tabular}{|c|c|c|c|c|c|c|c|c|c|c|}
\hline Year & ${ }^{\text {a Population }}(P)$ & Total Population & Guiyu NCSP & NMGD & MGDMPT & $\begin{array}{l}\text { Control } \\
{ }^{\mathrm{a}} \text { Population }\end{array}$ & NCSP & NMGD & MGDMPT & $\begin{array}{l}\text { P for Guiyu } \\
\text { vs. control }\end{array}$ \\
\hline 2001 & 120,080 & 220,080 & 57,333 & 237 & 1.077 & - & - & - & - & - \\
\hline 2002 & 132,378 & 232,378 & 61,479 & 226 & 0.973 & - & - & - & - & - \\
\hline 2003 & 133,727 & 233,727 & 57,230 & 271 & 1.159 & - & - & - & - & - \\
\hline 2004 & 134,741 & 234,741 & 53,307 & 331 & 1.410 & 406,874 & 113,729 & 164 & 0.403 & $<0.05$ \\
\hline 2005 & 136,417 & 236,417 & 52,800 & 126 & 0.533 & 409,900 & 107,269 & 158 & 0.385 & $<0.05$ \\
\hline 2006 & 138,355 & 238,355 & 55,872 & 59 & 0.248 & 412,171 & 106,564 & 117 & 0.284 & $>0.05$ \\
\hline 2007 & 141,227 & 241,227 & 50,608 & 117 & 0.485 & 414,608 & 108,592 & 81 & 0.195 & $<0.05$ \\
\hline 2008 & 143,988 & 243,988 & 47,009 & 270 & 1.107 & 419,143 & 114,193 & 114 & 0.272 & $<0.05$ \\
\hline 2009 & 145,537 & 245,537 & 38,300 & 182 & 0.741 & 423,200 & 118,417 & 248 & 0.586 & $<0.05$ \\
\hline Total & 122,6450 & 212,6450 & 473,938 & 1,819 & 0.855 & 248,5896 & 668,764 & 882 & 0.355 & $<0.05$ \\
\hline
\end{tabular}

Total Population for Guiyu is the addition of local resident population $P$ and annual immigrant estimated to be 100,000

$M G D M P T$ male genital diseases morbidity per thousand, NMGD number of male genital disorders, NCSP numbers of clinic service patients

${ }^{a}$ Shantou Municipal Bureau of Statistics: http://sttj.shantou.gov.cn/

The online version of the original article can be found at http://dx.doi.org/ 10.1007/s11356-013-2289-2.

$\mathrm{X} . \mathrm{Xu} \cdot \mathrm{Y}$. Zhang $\cdot$ T. A. Yekeen $\cdot$ Y. Li $\cdot$ B. Zhuang $\cdot$ X. Huo $(\triangle)$

Analytical Cytology Laboratory, Provincial Key Laboratory of

Infectious Diseases and Molecular Immunopathology, Shantou

University Medical College, 22 Xinling Rd,

Shantou 515041, Guangdong, People's Republic of China

e-mail: xhuo@stu.edu.cn

X. Xu

Department of Cell Biology and Genetics, Shantou University

Medical College, Shantou 515041, China 